\title{
Current efficiency in organic light-emitting diodes with a hole-injection layer
}

\author{
Hui Wang, ${ }^{1}$ Kevin P. Klubek, ${ }^{2,3}$ and C. W. Tang ${ }^{2, a)}$ \\ ${ }^{1}$ Department of Physics and Astronomy, University of Rochester, Rochester, New York 14627, USA \\ ${ }^{2}$ Department of Chemical Engineering, University of Rochester, Rochester, New York 14627, USA \\ ${ }^{3}$ Research Laboratories, Eastman Kodak Company, Rochester, New York 14650, USA
}

(Received 14 July 2008; accepted 17 August 2008; published online 5 September 2008)

\begin{abstract}
We have systematically investigated the effect of layer structures on the current efficiency of prototypical hole-injection layer (HIL)/hole-transport layer (HTL)/electron-transport layer (ETL) organic light-emitting diodes based on $4,4^{\prime}, 4^{\prime \prime}$-tris[ $N$-(3-methylphenyl)- $N$-phenylamino]triphenylamine (MTDATA) as the HIL, 4, $4^{\prime}$-bis[ $N$-(1-naphthyl)- $N$-phenylamino $]$ biphenyl (NPB) as the HTL, and tris(8-quinolinolato)aluminum (Alq) as the ETL. With bilayer devices, the current efficiency is limited by exciplex emissions in the case of MTDATA/Alq and quenching of Alq emissions by $\mathrm{NPB}^{+}$radical cations in NPB/Alq. The improved current efficiency in trilayer MTDATA/NPB/Alq devices can be attributed to a reduction in $\mathrm{NPB}^{+}$radical cations at the NPB/Alq interface and a strong electric field in the NPB layer. (c) 2008 American Institute of Physics.
\end{abstract}

[DOI: $10.1063 / 1.2978349$ ]

Since they first appeared in the $1980 \mathrm{~s},{ }^{1}$ organic lightemitting diodes (OLEDs) have received much attention due to their potential use as a full-color display technology. Although the efficiency and the operational life of OLEDs have vastly improved through intensive research effort, the mechanism underlying these key performance parameters are not well understood. The basic OLED device has a bilayer organic thin-film structure such as indium tin oxide (ITO)/4,4'-bis[ $N$-(1-naphthyl)- $N$-phenylamino $]$ biphenyl (NPB)/Alq/LiF/Al, where ITO is the anode and $\mathrm{LiF} / \mathrm{Al}$ is the cathode, and NPB and Alq are the hole-transport layer (HTL) and the electron-transport layer (ETL), respectively. During operation, the injected holes and electrons recombine at or near the HTL/ETL interface, producing electroluminescence (EL). It has been shown that much improved OLED performance can be realized using a HIL/HTL structure, where HIL is the "hole-injection" layer inserted between the anode and the HTL. For example, with $\mathrm{CuPc}^{2}$ as the HIL as in $\mathrm{CuPc} / \mathrm{NPB} / \mathrm{Alq}$ where Alq also functions as the emissive layer, long-lived OLEDs have been obtained. Another common HIL material is $4,4^{\prime}, 4^{\prime \prime}$-tris [ $N$-(3-methylphenyl)- $N$-phenylamino]triphenylamine (MTDATA), ${ }^{3}$ with which enhanced current efficiency and operational stability have been demonstrated. High-efficiency OLEDs have also been reported in various HIL/HTL configurations. ${ }^{4-8}$ Furthermore, low-voltage and high-efficiency OLEDs can be realized with a $p$-doped $\mathrm{HIL}^{9-11}$ in which the layer thickness can be readily adjusted for optimal light extraction. It has been suggested that the enhanced performance in HIL/HTL devices is due to a sequence of cascaded hole-injection barriers present in the HIL/HTL/ETL structure, which produces a "balanced" electron-hole recombination at the HTL/ETL interface. ${ }^{12,13}$ In this paper, we systematically investigate the effect of the HIL on OLED performance characteristics, specifically the cause for current efficiency enhancement.

Figure 1 shows the multilayer OLED structure and the molecular structures for the HIL (MTDATA), HTL (NPB),

${ }^{a)}$ Electronic mail: chtang@che.rochester.edu. and ETL (Alq). The Fabrication of OLEDs by physical vapor deposition is described elsewhere. ${ }^{14}$

Figure 2(a) shows the current density versus voltage $(J-V)$ characteristics for a series of OLED devices $A-F$. The inset table lists the HIL/HTL layer configurations along with the current efficiencies measured in candela/ampere. Common for all the devices is Alq as the ETL, which is $70 \mathrm{~nm}$ thick. Bilayer devices A and B utilize NPB and MTDATA, respectively, as the HTL. Devices C, D, E, and F have a trilayer structure with MTDATA as the HIL and NPB as the HTL. In bilayer devices A and B, because the hole mobility in NPB and MTDATA (Refs. 12, 15, and 16) is much higher than the electron mobility in Alq, the voltage drop in the HTL is negligible compared to that in Alq. In trilayer devices $\mathrm{C}-\mathrm{F}$, the energy offset between the highest occupied molecular orbital (HOMO) energy levels presents a hole-injection barrier of $0.4 \mathrm{eV}$ at the MTDATA/NPB interface, causing an accumulation of holes on the MTDATA side of the interface.
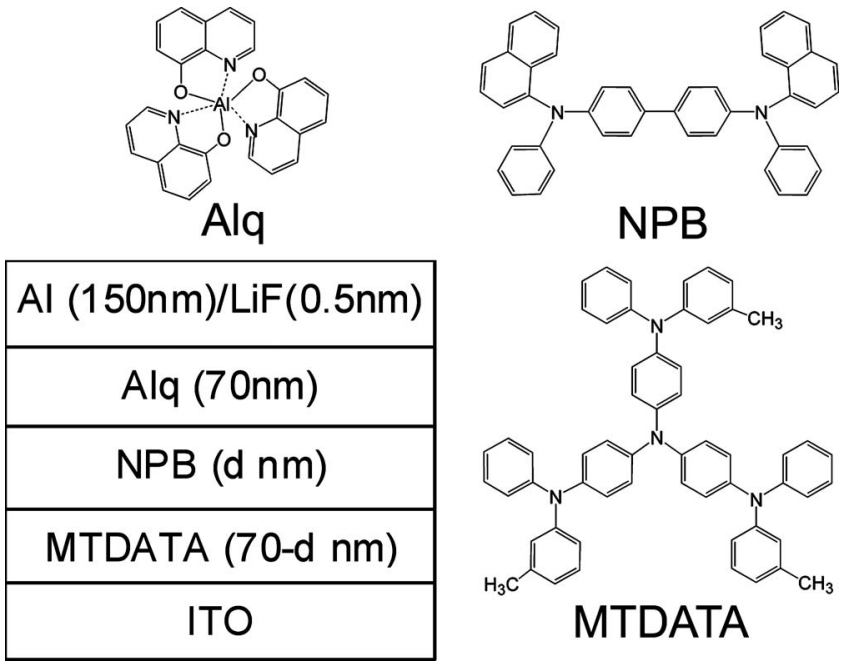

FIG. 1. Structure of OLEDs and materials used. HOMO energy level: MTDATA $(5.1 \mathrm{eV})$, NPB $(5.5 \mathrm{eV})$, and Alq $(5.7 \mathrm{eV}) . d$ is the variable thickness of the NPB layer. 

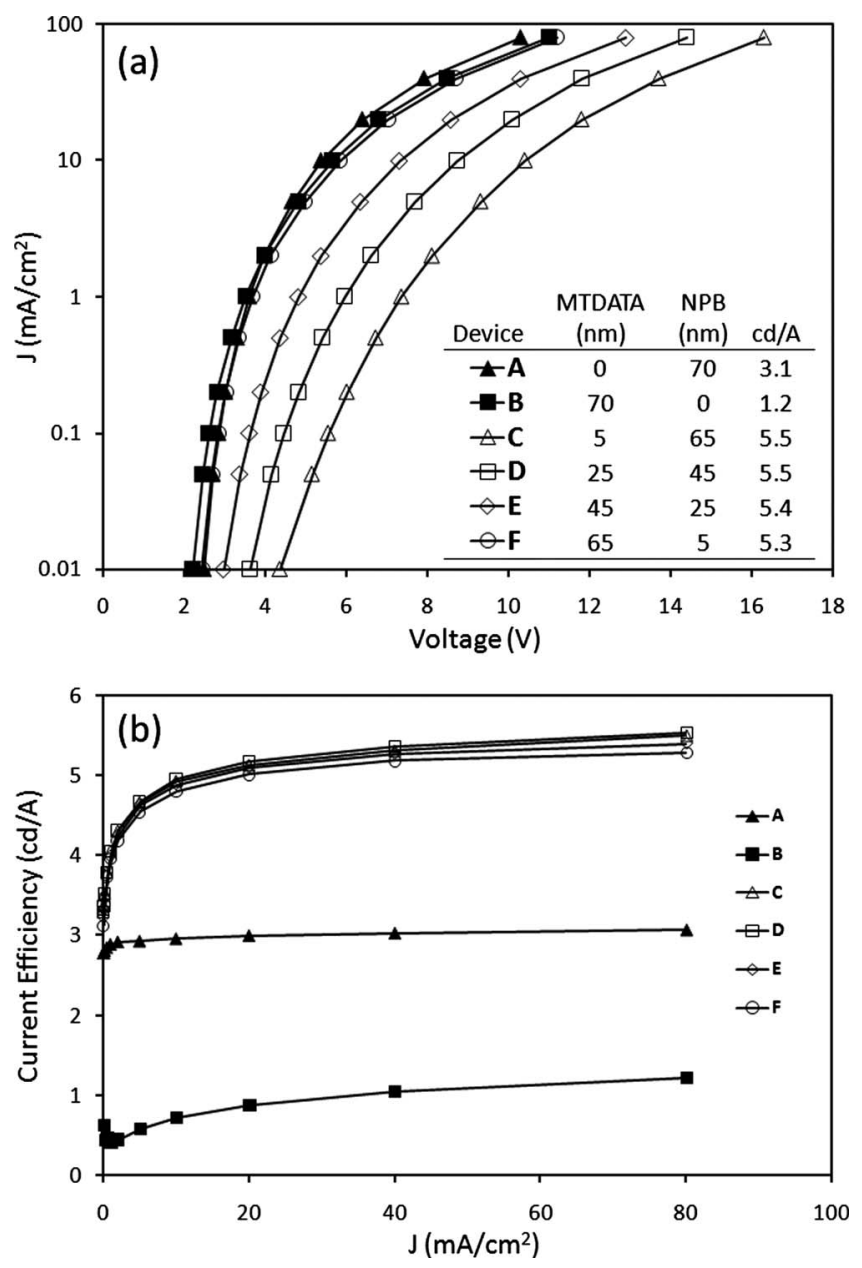

FIG. 2. (a) Current density $(J)$ vs voltage $(V)$ and (b) current efficiency vs $J$ for MTDATA/NPB/Alq devices with variable NPB layer thicknesses. Inset table: Layer thickness configurations and current efficiency at $80 \mathrm{~mA} / \mathrm{cm}^{2}$.

As a consequence, the voltage drop in the NPB layer is no longer negligible. This additional voltage drop in the NPB layer has manifested in a higher driving voltage for the trilayer devices in comparison with the bilayer devices, as evident from Fig. 2(a). Furthermore, for a given current density, the driving voltage increases almost linearly with the thickness of the NPB layer, as shown in Fig. 3, indicating that the electric field in the NPB layer is relatively constant. Assuming that the voltage drop in the MTDATA layer is negligible, the electric field in the NPB layer can be estimated according to Eq. (1),

$$
V=E_{\mathrm{NPB}}(J) \times d_{\mathrm{NPB}}+V_{\mathrm{Alq}}+\Delta V_{\mathrm{wf}},
$$

where $V$ is the driving voltage, $E_{\mathrm{NPB}}(J)$ is the electric field in the NPB layer under current density $J, d_{\mathrm{NPB}}$ is the thickness of the NPB layer, $V_{\text {Alq }}$ is the voltage drop in Alq which is assumed to be constant for a given current density, and $\Delta V_{\mathrm{wf}}$ $(\sim 1.45 \mathrm{~V})$ is the work function difference between the anode and the cathode. The slope of each line plotted in Fig. 3 is the electric field in the NPB layer, which increases from 0.45 to $0.84 \mathrm{MV} / \mathrm{cm}$, as the current density increases from 0.1 to $80 \mathrm{~mA} / \mathrm{cm}^{2}$. Figure 3 inset shows that the current efficiency increases linearly with the electric field in the NPB layer from 3.3 to $5.5 \mathrm{~cd} / \mathrm{A}$.

Figure 2(b) shows the current efficiency of devices A-F as a function of current density. The MTDATA/Alq bilayer

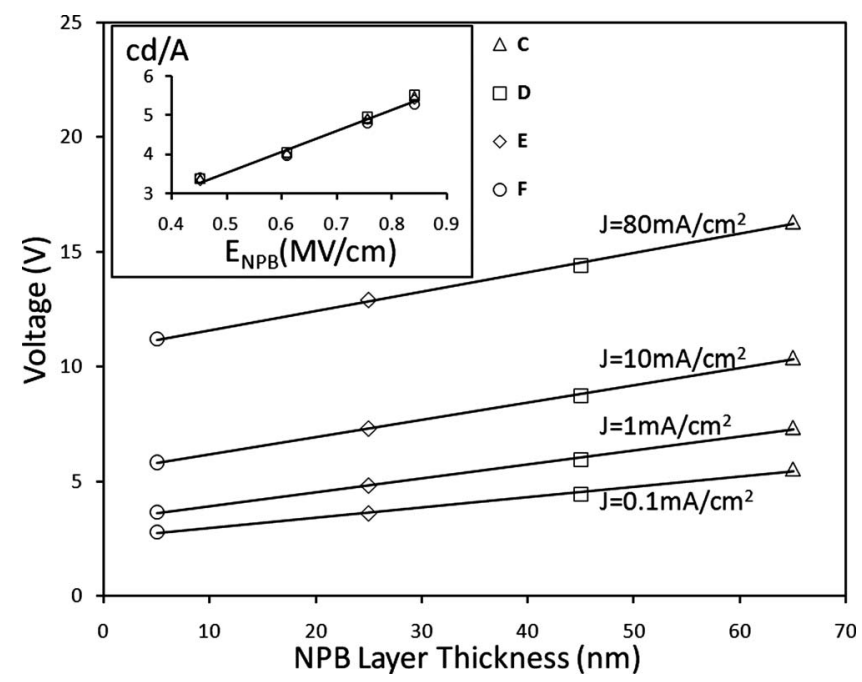

FIG. 3. Driving voltage vs NPB layer thickness of trilayer devices under current densities of $0.1,1,10$, and $80 \mathrm{~mA} / \mathrm{cm}^{2}$. Inset: current efficiency vs electric field in the NPB layer for the trilayer devices.

device $\mathrm{B}$ has the lowest current efficiency of $1.2 \mathrm{~cd} / \mathrm{A}$ (measured at $80 \mathrm{~mA} / \mathrm{cm} 2$ ) compared to $3.1 \mathrm{~cd} / \mathrm{A}$ for the NPB/Alq bilayer device $\mathrm{A}$, and $\sim 5.5 \mathrm{~cd} / \mathrm{A}$ for trilayer devices $\mathrm{C}-\mathrm{F}$. Also noted is the large percent increase in the current efficiency with current density for MTDATA/Alq bilayer device $\mathrm{B}$ and for trilayer devices C-F. In contrast, the current efficiency is relatively independent of the current density for the $\mathrm{NPB} / \mathrm{Alq}$ bilayer device A.

The low current efficiency for device B has been attributed to the formation of exciplex, ${ }^{13,17}$ at the MTDATA/Alq interface, where the EL emission quantum yield is controlled by the exciplex emission with a lower quantum yield than Alq. Being a strong donor, MTDATA has a strong propensity to form exciplex with the acceptor Alq. This device also exhibits a redshift in the EL spectrum relative to the Alq emission, which has been attributed to emission from the MTDATA-Alq exciplex. ${ }^{17}$ With increasing current density, the EL spectrum shows a gradual blueshift along with an increase in the current efficiency. This appears to indicate a decrease in exciplex emissions at the MTDATA/Alq interface in favor of Alq emissions due to increased hole injection into the bulk of Alq.

There is little evidence for exciplex formation in the NPB/Alq bilayer device A. The EL spectrum exhibits the characteristic Alq emission and, unlike the MTDATA/Alq device, it shows hardly any blueshift with current density of up to $80 \mathrm{~mA} / \mathrm{cm}^{2}$. The higher current efficiency in the NPB/ Alq device compared to the MTDATA/Alq device can be attributed to the lack of exciplex emissions, which is consistent with NPB being a weaker donor compared to MTDATA.

The current efficiency in trilayer devices $\mathrm{C}-\mathrm{F}$ is substantially improved over NPB/Alq bilayer device despite the fact that they have in common the NPB/Alq interface. It has been reported that charge quenching can play a significant role in determining the current efficiency of EL devices under various device configurations and drive conditions. ${ }^{14,18,19}$ It has been suggested that exciton quenching at the NPB/Alq interface due to the accumulation of $\mathrm{NPB}^{+}$radical cations ${ }^{20,21}$ at the interface largely determines the current efficiency. In the case of the bilayer NPB/Alq device, the electric field in the NPB layer is negligible under forward bias, and the NPB/Alq 
interface is acting as a virtual anode with $\mathrm{NPB}^{+}$(hole) density primarily determined by the electric field in the Alq layer. Substantial quenching can therefore take place if the conditions for Förster energy transfer from Alq excitons to $\mathrm{NPB}^{+}$radical cations are satisfied. Preliminary chargemodulation experiments, which will be reported elsewhere, have confirmed the role of $\mathrm{NPB}^{+}$as the likely quenchers in the NPB/Alq bilayer device.

In the MTDATA/NPB/Alq trilayer devices, an electric field is induced in the NPB layer due to the accumulation of holes at the MTDATA/NPB interface. The net surface charge density $\sigma$ at the NPB/Alq interface can be expressed as in Eq. (2),

$$
\sigma=\sigma_{h}-\sigma_{e}=\varepsilon_{r} \varepsilon_{0} \Delta E=\varepsilon_{r} \varepsilon_{0}\left(E_{\mathrm{Alq}}-E_{\mathrm{NPB}}\right),
$$

where $\sigma_{h}\left(\sigma_{e}\right)$ is the surface density of holes (electrons) at the NPB/Alq interface, $\varepsilon_{r}$ and $\varepsilon_{0}$ are the relative (which are supposed to be the same for NPB and Alq) and vacuum dielectric constants, and $E_{\mathrm{Alq}}$ and $E_{\mathrm{NPB}}$ are the electric fields in the Alq and NPB layer. $\sigma_{e}$ and $E_{\mathrm{Alq}}$ are presumably constant for a given current density through both bilayer and trilayer devices. Increasing $E_{\mathrm{NPB}}$ will cause $\sigma_{h}$ to decrease proportionally. Accordingly, charge quenching due to $\sigma_{h}$ is reduced and the current efficiency is increased. Under a given current density, $E_{\mathrm{NPB}}$ is approximately the same for all the trilayer devices (Fig. 3), and the current efficiency, which is proportional to $E_{\mathrm{NPB}}$ as shown in the inset of Fig. 3, is independent of the NPB layer thickness.

It can be seen in Fig. 2 that the current efficiency for the MTDATA/NPB/Alq trilayer devices increases with increasing current density. As in the case of the MTDATA/Alq device, this can be explained in terms of enhanced hole injection and bulk recombination in the Alq layer at a higher electric field in the NPB layer, while reducing recombination at the NPB/Alq interface.

In conclusion, we have shown that the enhanced current efficiency observed in OLEDs with a trilayer MTDATA/ $\mathrm{NPB} / \mathrm{Alq}$ structure is due to the presence of a hole-injection barrier at the MTDATA/NPB interface, which creates an electric field in the NPB layer and reduces the hole density at the NPB/Alq interface. $\mathrm{NPB}^{+}$radical cations have been attributed as quenchers of Alq emissions.

We thank Rebecca Winter for device fabrication and Eastman Kodak for funding support.

${ }^{1}$ C. W. Tang and S. A. Vanslyke, Appl. Phys. Lett. 51, 913 (1987).

${ }^{2}$ S. A. VanSlyke, C. H. Chen, and C. W. Tang, Appl. Phys. Lett. 69, 2160 (1996).

${ }^{3}$ Y. Shirota, Y. Kuwabara, H. Inada, T. Wakimoto, H. Nakada, Y. Yonemoto, S. Kawami, and K. Imai, Appl. Phys. Lett. 65, 807 (1994).

${ }^{4}$ Z. B. Deng, X. M. Ding, S. T. Lee, and W. A. Gambling, Appl. Phys. Lett. 74, 2227 (1999).

${ }^{5}$ H. Jiang, Y. Zhou, B. S. Ooi, Y. Chen, T. Wee, Y. L. Lam, J. Huang, and S. Liu, Thin Solid Films 363, 25 (2000).

${ }^{6}$ D. Liu, C. G. Zhen, X. S. Wang, D. C. Zou, B. W. Zhang, and Y. Cao, Synth. Met. 146, 85 (2004).

${ }^{7}$ S. F. Chen and C. W. Wang, Appl. Phys. Lett. 85, 765 (2004).

${ }^{8}$ J. Li, C. Ma, J. Tang, C. S. Lee, and S. Lee, Chem. Mater. 17, 615 (2005).

${ }^{9}$ X. Zhou, M. Pfeiffer, J. Blochwitz, A. Werner, A. Nollau, T. Fritz, and K. Leo, Appl. Phys. Lett. 78, 410 (2001).

${ }^{10}$ J. S. Huang, M. Pfeiffer, A. Werner, J. Blochwitz, K. Leo, and S. Y. Liu, Appl. Phys. Lett. 80, 139 (2002)

${ }^{11}$ G. He, O. Schneider, D. Qin, X. Zhou, M. Pfeiffer, and K. Leo, J. Appl. Phys. 95, 5773 (2004).

${ }^{12}$ J. Staudigel, M. Stossel, F. Steuber, and J. Simmerer, J. Appl. Phys. 86, 3895 (1999).

${ }^{13}$ C. Giebeler, H. Antoniadis, D. D. C. Bradley, and Y. Shirota, J. Appl. Phys. 85, 608 (1999)

${ }^{14}$ D. Y. Kondakov, J. R. Sandifer, C. W. Tang, and R. H. Young, J. Appl. Phys. 93, 1108 (2003).

${ }^{15}$ S. Naka, H. Okada, H. Onnagawa, Y. Yamaguchi, and T. Tsutsui, Synth. Met. 111-112, 331 (2000).

${ }^{16}$ J. U. Wallace, R. H. Young, C. W. Tang, and S. H. Chen, Appl. Phys. Lett. 91, 152104 (2007).

${ }^{17}$ K. Itano, H. Ogawa, and Y. Shirota, Appl. Phys. Lett. 72, 636 (1998).

${ }^{18}$ R. H. Young, C. W. Tang, and A. P. Marchetti, Appl. Phys. Lett. 80, 874 (2002).

${ }^{19}$ T. Haskins, A. Chowdhury, R. H. Young, J. R. Lenhard, A. P. Marchetti, and L. J. Rothberg, Chem. Mater. 16, 4675 (2004).

${ }^{20}$ M. Sims, S. W. Venter, and I. D. Parker, SID Int. Symp. Digest Tech. Papers 2008, 223.

${ }^{21}$ R. H. Young, J. R. Lenhard, D. Y. Kondakov, and T. K. Hatwar, SID Int. Symp. Digest Tech. Papers 2008, 705. 